\title{
Deltopectoral Flap Reconstruction of the Posterior Pharyngeal Wall: A Single Stage Pedicle Flap Alternative Solution to the Free Flap Reconstruction of Circumferential Laryngopharyngeal Defects
}

\author{
Swee Keong Kang ${ }^{1}$ Sabih Nadeem Qamar $^{1} \cdot$ Theofano Tikka $^{1}$ (I) \\ Thomas Daniel Milner ${ }^{1}$
}

Received: 21 August 2021 / Accepted: 28 November 2021 / Published online: 7 January 2022

(C) Association of Otolaryngologists of India 2021

\begin{abstract}
The first option for reconstruction of a circumferential pharyngeal defect following extensive pharyngolaryngectomy is free tissue transfer. Despite that, pedicled flaps can be used when microsurgical expertise is not available or if other patient related or region related issues deem free tissue transfer unfavourable. The aim of this study was to review the operative feasibility and functional outcomes following dual flap reconstruction of circumferential pharyngeal defects. This was a retrospective study of all patients $(\mathrm{n}=8)$ who underwent either primary $(\mathrm{n}=5)$ or salvage $(\mathrm{n}=3)$ circumferential laryngopharyngectomy $+/-$ cervical oesophagectomy, followed by dual flap reconstruction, with a deltopectoral flap to reconstruct the posterior wall from 2005 to 2020. The main outcome measures were operative complications, hospital stay and functional outcomes (speech and swallowing). The operation was feasible in all patients, with dual flap reconstruction using a deltopectoral flap, combined with a pectoralis major flap $(\mathrm{n}=5)$ or a supraclavicular flap $(\mathrm{n}=3)$. All patients developed a small, lateralised, selfhealing fistula at the site of the deltopectoral flap 3-point junction. This did not require any intervention, or impact on adjuvant treatment. Functional outcomes were favourable, with all patients achieving oral diet. One patient required gastrostomy diet supplementation, and one patient required stricture dilatation. Of the patients able to receive a speech valve $(n=4)$, all achieved intelligible speech. Dual flap reconstruction of circumferential pharyngeal defects represents a feasible alternative option for a
\end{abstract}

Theofano Tikka

Theofano.tikka@gmail.com

1 Department of Otolaryngology, Head and Neck Surgery, Monklands University Hospital, Airdre, Scotland complex reconstructive problem. The predictable operative recovery and favourable functional outcomes indicate that the use of both a deltopectoral flap and a second flap is a robust reconstructive solution.

Keywords Pedicled flap · Pharyngectomy . Laryngectomy · Case series · Deltopectoral · Pectoralis major myocutaneous flap

\section{Introduction}

Circumferential laryngopharyngectomy and cervical oesophagectomy present a unique reconstructive challenge due to the complete loss of both the anterior and posterior pharyngeal walls. The radial forearm free flap (RFFF), the latissimus dorsi flap, the anterolateral thigh flap, the free jejunal flap (FJF) and the pectoralis major myocutaneous flap (PMMC) are more commonly described in the literature for reconstruction used either tubed or sutured in a 'horseshoe-shaped' fashion to the prevertebral fascia. [1] In the early surgical series, the use of free flaps was associated with a higher rate of post-operative fistula formation (up to $67 \%$ ) compared to PMMC flap reconstruction $(22 \%)[2,3]$ but the percentage of such complications in the former groups has significantly improved in more recent studies (11-14\% fistula;14-16\% stenosis). [4] However, the biggest challenge is regarding the long-term functional outcomes of swallow and speech, which remain poor and difficult to produce good outcomes consistently.

Despite the fact that free tissue transfer remains the first option for reconstruction of circumferential pharyngeal defects, pedicled flaps are a valid alternative in regions where there is no availability of microsurgical team. Over the past 18 months, many head and neck units have opted 
to reconstruct oncological defects using pedicled flaps to shorten operative time and the need for post-operative intensive care unit to allow patients to proceed with their operations and reconstruction despite the scarce hospital resources during the covid-19 pandemic. [5, 6] During the first wave of the pandemic some units completely stopped performing free flap reconstruction and opted for pedicled and/or staged defect reconstruction. [7] During the Covid19 pandemic, we have continued to perform laryngectomies with pedicled flap reconstruction as needed following the peri-operative protocol recommended by the British Association of Head and Neck Oncologist [8], we have also published our local hospital peri-operative protocol and our experience with the management of a patient found to be covid-19 positive during the pre-operative assessment stage. This required postponing the procedure until he was negative. [9]

In our unit, it was common practice prior to the pandemic to reconstruct the circumferential pharyngeal defects with a single staged procedure using a deltopectoral flap for reconstruction of the posterior pharyngeal wall and another flap (PMMC or supraclavicular flap in our case series) to complete the anterior reconstruction of the circumferential pharyngo-oesophageal defects. This is the first time that this single stage approach is described in the literature. In this paper, we are presenting our experience with the technique, including a detailed presentation of the surgical steps and our surgical outcomes.

\section{Methods}

\section{Patient Population}

A retrospective case series review was conducted in the head and neck unit of a district general hospital, from January 2005 (when the senior author SK started his consultancy post), until December 2020. This identified all patients who had undergone circumferential laryngopharyngectomy \pm cervical oesophagectomy with dual flap reconstruction using the deltopectoral (DP) flap to reconstruct the posterior wall and another flap to cover the defect anterolaterally. Eight patients were identified during the study period.

\section{Ethical Considerations}

Institutional approval was attained following discussion at the local Head and Neck Oncology multidisciplinary team meeting and Caldicott Guardian approval was granted.

\section{Surgical Technique}

Reconstruction is performed following laryngectomy and circumferential pharyngectomy $+/$ - cervical oesophagectomy. A DP flap is raised in the subfascial plane. The blood supply from the 2 nd and 3rd internal mammary perforator arteries allows harvesting of a broad tissue flap with a width extending from the clavicle to the 5th intercostal space, and a length reliably extending towards the shoulder tip. The flap is sutured onto the posterior oropharyngeal mucosa superiorly, and the proximal oesophagus inferiorly (Fig. 1). In one individual, the superior extent of the flap repair extended to the level of the soft palate. This individual had a short and broad neck that allowed the DP flap to reach the soft palate. Nevertheless, this can be challenging in patients with long neck or in cachectic patients with poor tissue quality. In these occasions or when the resection is so extensive to require removal of tissue up to the level of the soft palate, there is no need to over-stretch the DP flap to reach and be sutured to the soft palate line. It is important for the DP flap to reach the tongue base and good closure to be achieved at the lateral superior part of the flap to the tongue base. This will prevent saliva leak at this point and create the broad funnel needed for direction of the saliva via the neopharynx to the oesophagus without causing stenosis. The superior edge of the posterior pharyngeal wall can be left to heal by granulation in the longnecked patients that the DP flap cannot completely reach the mucosa line. This modification is rarely required but should be mentioned as it can accommodate for anatomical variations and differences in the extend of cancer requiring resection. Total time required to raise the DP flap and suture it to the inferior edge of the oropharynx is approximately $30 \mathrm{~min}$.

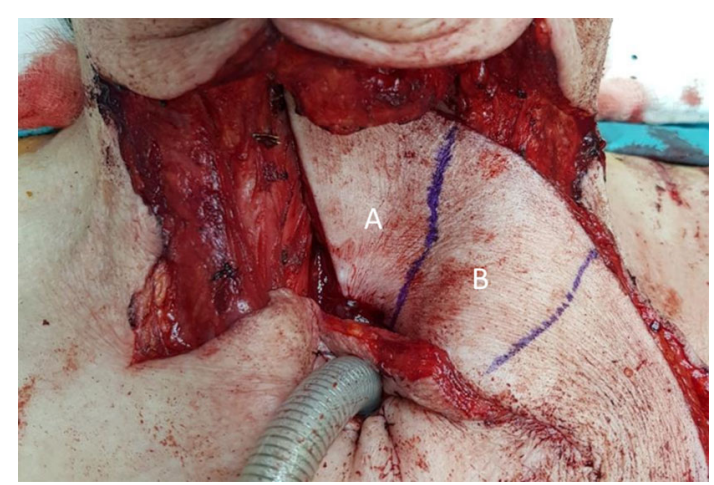

Fig. 1 DP flap raised and sutured into position proximally and distally. Note the conical shape of the neopharynx generated naturally upon positioning the DP flap. A Region of DP flap forming neopharynx. B Region of DP flap to be de-epithelialise 
A second flap is then harvested. This can be a free flap or a pedicled flap. In our series, a PMMC flap or a supraclavicular fasciocutaneous flap on the opposite side was used. This is to form the anterior and lateral pharyngeal wall. This second flap is raised and inserted in a similar manner to a partial pharyngeal patch repair. [3]

The distal part of the deltopectoral cutaneous tissue is left to form the posterior pharyngeal wall and skin deepithelialisation is performed to a small middle segment of the flap, so that the only cutaneous tissue buried within the neck is the neopharynx itself (Fig. 2). The skin of the deltopectoral flap near the stump of the oesophagus is sutured, thereby ensuring the integrity of the neo-posterior pharyngeal wall from oropharynx to oesophagus.

The second flap can then be sutured to the anterior mucosa of the proximal oesophagus, the DP flap laterally, and the tongue base superiorly, creating a conical neopharynx. Flap harvest sites are then closed primarily. A salivary bypass tube is positioned in the reconstructed neopharynx to help the healing process splinting it open, which is subsequently removed 3 weeks later, prior to commencement of oral diet. Six-month post-operative outcome is displayed in Fig. 3.

\section{Results}

The case series includes 8 patients (Male:Female $=4: 4$ with a mean age of 68.6 years (range 55-82). Five procedures were performed as primary circumferential laryngopharyngectomies, and three patients underwent salvage operations following previous radical chemoradiotherapy (7 years, 9 years and 28 years prior to surgery). Primary tumour maximal diameters ranged from $17-75 \mathrm{~mm}$ (mean: $41.6 \mathrm{~mm}$ ).

The anterior pharyngeal wall was reconstructed with a PMMC flap for 5 patients and a supraclavicular flap for the remaining 3 patients. All patients developed a small

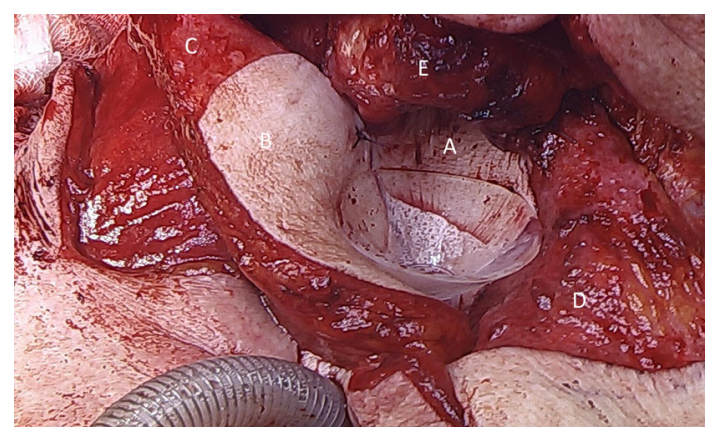

Fig. 2 Neopharynx being created from the DP flap A and SC flap $\mathbf{B}$ around a salivary bypass tube. Laryngeal stoma is notable inferiorly. C De-epithelialised SC flap. D De-epithelialised DP flap. E Pharyngeal remnant

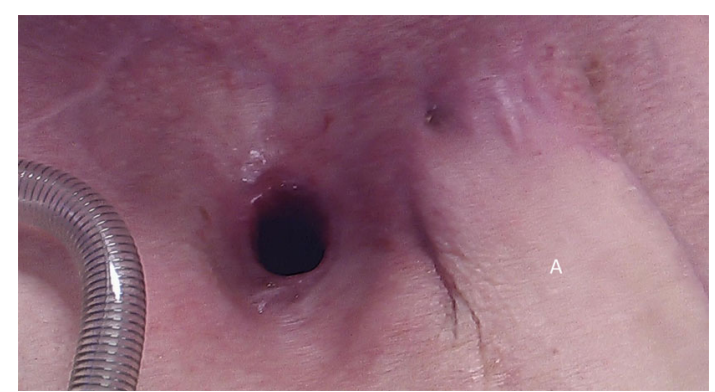

Fig. 3 Post-operative outcome - an external view of the neck and stoma. A Deltopectoral flap

pharyngocutaneous fistula, laterally in the neck, over the 3-point junction of the DP flap. In all patients this required no intervention, did not result in wound dehiscence, and closed spontaneously in a mean of 15 days post-operatively. For the three patients requiring adjuvant (chemo)radiotherapy, there was no delay in treatment delivery.

Functional outcomes were favourable in the majority of patients. All patients have achieved normal $(n=2)$ or soft $(n=6)$ diet, although one individual continues to require PEG tube dietary supplementation. Of the 6 patients that manage soft diet, one developed a low neopharyngeal stricture requiring repeated dilatations. A speech valve has been inserted in 4 patients, with all achieving intelligible speech, 2 patients are awaiting tracheo-oeosophageal puncture which has been postponed during the COVID-19 pandemic. Speech valve insertion was not possible for 2 patients, as the oesophagectomy level is significantly below the level of the stoma. Attempt of insertion of a valve when the oesophageal segment is low can compromise stoma and flap reconstruction healing as the valve can cause tension around the flap and stoma reconstruction. The PMMC is very bulky, so no attempt is made to insert the valve through the PMMC. During the months to follow, the PMMC starts to atrophy and become less bulky, and it is due to this process that our patients achieve good long term voice outcomes. Two patients died during follow up, one following tumour recurrence, and one unrelated to their malignancy. All results are summarised in Table 1.

\section{Discussion}

This case series presents an alternative method for reconstruction of circumferential pharyngeal defects using a dual flap technique, with the rotated deltopectoral flap as the posterior wall of the neopharynx. This technique reconstructs the neopharynx with minimal soft tissue bulk in the central compartment space, creating a wider conduit for food passage. A single tubed flap, with the exception of the 


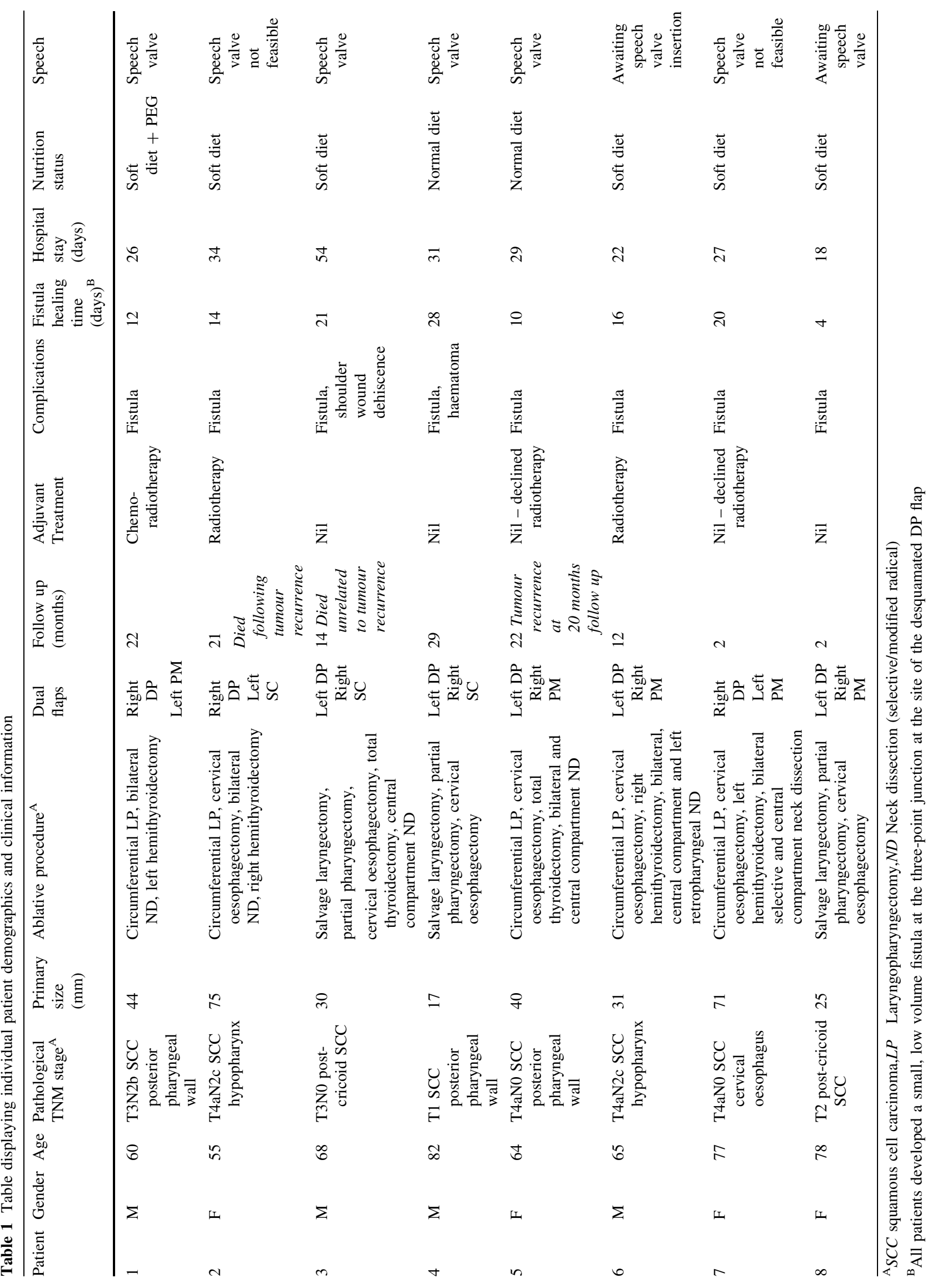


jejunal free flap, incorporates all the flap bulk in the central compartment, limiting the calibre of the neopharynx. Positioning of the dual flaps resulted in a conical shaped neopharynx (Fig. 2), resembling the natural pharynx more than a tubed flap reconstruction. The authors theorise that this wider, conical neopharynx would both enhance the swallow function, and allow improved air passage for speech. Our limited series seems to support this, with good functional outcomes reported.

The additional advantage to the DP flap, is that it can be rapidly raised and positioned on the posterior wall, requiring minimal de-epithelialisation. This also converts a complex circumferential defect into a 'partial' defect, and consequently, does not contribute significantly to the surgical time. Of course, the surgical time to perform the reconstruction can prove to be longer for the novice surgeon as there is an expected learning curve associated with harvesting and placing the flap, appreciating and taking into consideration the size of the defect that needs to be covered and the quality and characteristics of the surrounding skin and tissues to allow a successful reconstruction. [10].

To the authors knowledge, the combination of a DP and a second flap has never been described before in the literature, nevertheless, the DP flap has been used before to reconstruct the posterior pharyngeal wall in 2 stages procedures from a surgical series reported from the head and neck team in Hong Kong. [11] Various other techniques have been described for reconstructing circumferential defects, including tubed free flaps, tubed pedicled flaps, and suturing of flaps onto the prevertebral fascia. [1, 12] A recent multicentre study concluded that the type of flap used during reconstruction does not impact on the postoperative swallowing outcomes with just over half of patients being able to gain normal diet post-operatively (54\% with any flaps; 63\% RFFF. 53\% PMMC, 58\% FJF) with no significant difference between the different flaps being used. [1] Other series have demonstrated superior functional outcomes when free flaps were used $(58 \%-$ $63 \%$ adequate oral nutrition; $21-25 \%$ stricture rate) compared to tubed PMMC or PMMC with the prevertebral fascia forming the posterior pharyngeal wall (53-91\% adequate oral nutrition; $18-40 \%$ stricture rate; $43 \%$ satisfactory vocal function). [13-16] In our case series, all patients ultimately achieved oral diet intake, with 7 patients achieving sufficient intake to support their complete nutrition. Only one patient developed a stricture requiring repeated dilatation. All patients who were able to receive a tracheooesophageal puncture attained intelligible speech.

The main weakness of the dual flap technique lies in the likelihood of patients developing a small fistula. This occurs at the de-epithelialised region on the DP flap. While an important consideration in the perioperative period, this has not impacted on subsequent care or adjuvant treatment delivery in any of the patients. Tissue handling is very important during the dual flap reconstruction to ensure that the blood supply to the flaps will not get compromised. This is particularly the case for the fasciocutaneous DP flap. To achieve this, gentle suturing of the junction between the pectoralis major flap and the de-epithelialised segment of the DP flap is of paramount importance. Care should be taken to ensure that the suture line is not too tight in that area, this can make the closure not completely water-tight hence resulting in a minor leak in this segment but on the other hand it ensures the viability of the flap. Overjealous tightening of the suture line can compromise the blood supply to the distal end of the DP flap and strangulate it. The fistula has always remained small and low volume, requiring minimal/no intervention, and the authors are of the opinion that the long-term functional outcomes outweigh the consequences of fistula development. In fact, the small fistula may be advantageous, allowing a means of controlled drainage, as none of the patients have developed significant tissue breakdown. The use of the salivary bypass tube helps to minimise the amount of resultant leak, expediting the healing of the fistula site. The factors that pre-dispose to fistulae have been investigated extensively previously, including comparing between free and pedicled flaps. [17] Despite a recent trend towards increasing free flap use, morbidity profiles appear to be similar between free and pedicled flaps. ${ }^{17}$ Although all patients in our case series developed a fistula, the short-lived nature, and the rapid healing indicate that dual flap use appears to be a robust reconstruction technique.

\section{Conclusions}

Dual DP/second flap reconstruction of circumferential pharyngeal defects represents a single stage pedicle flaps reconstruction solution to a complex reconstructive problem, with the DP flap converting the circumferential defect into a 'partial' defect. This can be considered in areas where there is no availability of microsurgical team or during health crisis like the current covid-19 pandemic. Our series demonstrated the reliability of this technique in providing reasonable quality of life to patients. The authors recommend this as an alternative to the single pedicled or free flap tubed reconstruction for large circumferential pharyngeal defects.

\section{Declarations}

Conflict of interest The authors declare that they have no conflict of interest. 


\section{References}

1. Morinière S, Gorphe P, Espitalier F, Blanchard D, Fakhry N, Saroul N et al (2019) Assessment of swallowing function after circumferential pharyngolaryngectomy. A multicenter study by the GETTEC group. Eur Ann Otorhinolaryngol Head Neck Dis 136(1):3-5

2. Kelly KE, Anthony JP, Singer M (1994) Pharyngoesophageal reconstruction using the radial forearm fasciocutaneous free flap: preliminary results. Otolaryngol Head Neck Surg 111(1):16-24

3. Spriano G, Pellini R, Roselli R (2002) Pectoralis major myocutaneous flap for hypopharyngeal reconstruction. Plast Reconstr Surg 110(6):1408-1413

4. Reiter M, Baumeister P (2019) Reconstruction of laryngopharyngectomy defects: comparison between the supraclavicular artery island flap, the radial forearm flap, and the anterolateral thigh flap. Microsurgery 39(4):310-315

5. Day AT, Sher DJ, Lee RC, Truelson JM, Myers LL, Sumer BD et al (2020) Head and Neck oncology during the COVID-19 pandemic: reconsidering traditional treatment paradigms in light of new surgical and other multilevel risks. Oral Oncol 105: 104684

6. Desai SC, Seth R (2020) Shifting paradigm in head and neck reconstructive surgery during the COVID-19 crisis. Facial Plast Surg Aesthet Me 22:152-154

7. Patel RJ, Kejner A, McCullen C (2020) Early institutional head and neck oncologic and microvascular surgery practice patterns across the United States during the SARS-CoV-2 (COVID19) pandemic. Head Neck 42:1168-1172

8. BAHNO laryngectomy guidance during COVID-19 pandemic. In: https://www.bahno.org.uk/bahno_laryngectomy_guideance_ during_covid-19_pandemic.aspx [1 October 2021]

9. Coleman H, Tikka T, Okhovat S, Kang SK (2020) Planning considerations prior to laryngectomy for a patient infected with severe acute respiratory syndrome coronavirus- 2 pre-operatively [published online ahead of print 2020 Nov 4]. J Laryngol Otol 134(11):1029-1032

10. Gray ML, Drake VE, Desai SC (2021) Resurgence of regional flaps for head and neck reconstruction. Current Opin Otolaryngol Head Neck Surg. https://doi.org/10.1097/MOO.00000000000 000725

11. Chain RCL, Chan JYW (2014) Deltopectoral flap in the era of microsurgery. Surg Res Practice. https://doi.org/10.1155/2014/ 420892

12. Chu YH, Lai WS, Lin YY, Liu SC, Lee JC (2020) Pharyngeal reconstruction using a U-shaped pectoralis major myocutaneous flap: an effective technique that should not be forgotten. Eur Arch Otorhinolaryngol 277(1):217-220

13. Mura F, Bertino G, Occhini A, Mevio N, Scelsi D, Benazzo M (2012) Advanced carcinoma of the hypopharynx: functional results after circumferential pharyngolaryngectomy with flap reconstruction. Acta Otorhinolaryngol Ital 32(3):154-157

14. Espitalier F, Ferron C, Leux C, Jégoux F, Durand N, Beauvillain de Montreuil C et al (2012) Results after u-shaped pectoralis major myocutaneous flap reconstruction of circumferential pharyngeal defects. Laryngoscope 122(12):2677-2682

15. Burke MS, Kaplan SE, Kaplowitz LJ, Lotempio MM, Hicks WL Jr, Rigual NR et al (2013) Pectoralis major myocutaneous flap for reconstruction of circumferential pharyngeal defects. Ann Plast Surg 71(6):649-651

16. Chan YW, Ng RW, Liu LH, Chung HP, Wei WI (2011) Reconstruction of circumferential pharyngeal defects after tumour resection: reference or preference. J Plast Reconstr Aesthet Surg 64(8):1022-1028

17. Sayles M, Grant DG (2014) Preventing pharyngo-cutaneous fistula in total laryngectomy: a systematic review and meta-analysis. Laryngoscope 124(5):1150-1163

Publisher's Note Springer Nature remains neutral with regard to jurisdictional claims in published maps and institutional affiliations. 